\title{
Effects of Simultaneously Performed Dual-Task Training with Aerobic Exercise and Working Memory Training on Cognitive Functions and Neural Systems in the Elderly
}

\author{
Hikaru Takeuchi $\mathbb{D}^{1},{ }^{1}$ Daniele Magistro ${ }^{(D},{ }^{2}$ Yuka Kotozaki, ${ }^{3}$ Kosuke Motoki, ${ }^{4}$ \\ Keyvan Kashkouli Nejad, ${ }^{4}$ Rui Nouchi, ${ }^{5,6,7}$ Hyeonjeong Jeong, ${ }^{4,8}$ Chiho Sato, ${ }^{4,9}$ \\ Salvatore Sessa, ${ }^{10}$ Ryoichi Nagatomi, ${ }^{11}$ Massimiliano Zecca $\mathbb{D}^{12,13}$ Atsuo Takanishi, ${ }^{14,15}$ \\ and Ryuta Kawashima ${ }^{1,7}$
}

${ }^{1}$ Division of Developmental Cognitive Neuroscience, Institute of Development, Aging and Cancer, Tohoku University, Sendai, Japan ${ }^{2}$ Department of Sport Science, School of Science and Technology, Nottingham Trent University, Nottingham, UK

${ }^{3}$ Division of Clinical research, Medical-Industry Translational Research Center, Fukushima Medical University School of Medicine, Fukushima, Japan

${ }^{4}$ Department of Functional Brain Imaging, Institute of Development, Aging and Cancer, Tohoku University, Sendai, Japan

${ }^{5}$ Creative Interdisciplinary Research Division, Frontier Research Institute for Interdisciplinary Science, Tohoku University, Sendai, Japan

${ }^{6}$ Human and Social Response Research Division, International Research Institute of Disaster Science, Tohoku University, Sendai, Japan

${ }^{7}$ Smart Ageing International Research Center, Institute of Development, Aging and Cancer, Tohoku University, Sendai, Japan

${ }^{8}$ Graduate School of International Cultural Studies, Tohoku University, Sendai, Japan

${ }^{9}$ Department of Surgical Oncology, Tohoku University School of Medicine, Sendai, Japan

${ }^{10}$ Graduate School of Creative Science and Engineering, Waseda University, Tokyo, Japan

${ }^{11}$ Division of Biomedical Engineering for Health and Welfare, Tohoku University Graduate School of Biomedical Engineering, Sendai, Japan

${ }^{12}$ National Centre for Sports and Exercise Medicine-East Midlands, Loughborough University, UK

${ }^{13}$ Wolfson School of Mechanical, Electrical and Manufacturing Engineering, Loughborough University, Loughborough, UK

${ }^{14}$ Department of Modern Mechanical Engineering, Waseda University, Tokyo, Japan

${ }^{15}$ Humanoid Robotics Institute, Waseda University, Tokyo, Japan

Correspondence should be addressed to Hikaru Takeuchi; takehi@idac.tohoku.ac.jp

Received 23 October 2019; Revised 29 March 2020; Accepted 23 May 2020; Published 5 June 2020

Academic Editor: Zygmunt Galdzicki

Copyright (C) 2020 Hikaru Takeuchi et al. This is an open access article distributed under the Creative Commons Attribution License, which permits unrestricted use, distribution, and reproduction in any medium, provided the original work is properly cited.

Working memory (WM) training (WMT), aerobic exercise training (AET), and dual-task training improve cognitive functions and alter neural systems in older adults. In particular, the effects have been investigated of dual-task training that combines a walking or standing activity (balance exercise) simultaneously performed with cognitive training (which is ecologically difficult for the elderly). In this study, we investigated the effects of simultaneously performed dual-task training incorporating both AET and WMT (SDAEWMT), using a recumbent ergocycle bicycle and a WMT program that provided a portable console and made the training ecologically easy for the elderly. Older adults $(65.9 \pm 13.7$ years old) participated in 3 months of SDAEWMT, WMT, or AET after random allocation, and the effects of SDAEWMT were compared with those of WMT and AET. Prior to and after training, all the subjects underwent cognitive testing, magnetic resonance imaging (MRI) involving diffusion tensor imaging (DTI), and functional MRI during performance of an N-back WM task. SDAEWMT improved executive function (performance of a frontal assessment battery); however, there was no evidence of broader transfer effects or enhanced learning with WMT. SDAEWMT resulted in mean diffusivity changes in brain areas involving the dopaminergic system, suggesting that neural tissue 
changes occurred in these areas. SDAEWMT also resulted in an increase in brain activity during the 2-back working memory task in brain areas involved in attentional reorientation. These results suggest that SDAEWMT is effective for improving cognitive functions and inducing beneficial neural changes in older adults.

\section{Introduction}

The proportion of older individuals is increasing worldwide in parallel with improvements in the average life expectancy [1]. Despite an increase in life expectancy, various studies have highlighted that aging is associated with deterioration in both physical and cognitive functions [2]. With regard to the cognitive aspects of aging, previous studies have shown changes in brain structure and function with advancing age [3], particularly in the prefrontal areas involved in executive function and the attention system [4] as well as in areas involved in working memory [5]. Therefore, it is important to identify and implement effective strategies and activities that can maintain and even improve the cognitive abilities of older adults. Cognitive training and physical training have been primarily investigated for this purpose. The combination of these approaches is the topic of the present study.

Working memory (WM) is the limited-capacity storage system involved in the maintenance and manipulation of information over a short time period [6]. Regions in the external attention system (EAS) that play a key role in external attention [7-9], such as the lateral prefrontal cortex (LPFC) and the posterior parietal cortex, are involved with WM and are activated during WM performance [6]. Dopaminergic transmission plays an important role in WM. Cortical dopamine release has been observed during WM [10], and dopaminergic neurotransmission and its dose are critical in WM performance and in the tuning of prefrontal activity during WM. A number of studies on WM training (WMT) have been conducted, and results are inconsistent; however, meta-analyses have shown that WMT improves the performance of untrained WM tasks as well as attention/inhibition [11] and nonverbal reasoning [12, 13], and the observed inconsistency could be due to methodological differences, as well as to statistical fluctuations and lack of statistical power in individual studies that were then combined with weak effects $[12,13]$. Such training also changes the brain activity patterns observed during the performance of WMrelated tasks and alters gray matter and white matter structures, particularly those within lateral frontal and lateral parietal areas, which are activated during WM tasks $[14,15]$. Furthermore, changes in the dopamine D1 receptor density in the lateral frontal and lateral parietal areas are seen after WMT [16]. WMT that involves updating was shown to reduce transient changes in the binding potentials of D2 receptors in the striatum during updating, which in turn was suggested to reflect increases in striatal dopamine release during the updating [17].

However, the extent of cognitive function plasticity subject to cognitive training is characterized by a low effect size $[11,12]$. Furthermore, the potential magnitude of these changes in cognitive function and neural changes resulting from cognitive training deteriorates with increasing age [14].
Aerobic exercise training (AET) is another approach for countering age-related cognitive decline. Numerous studies have been conducted on AET, and results are inconsistent; however, meta-analyses have shown that AET improves various cognitive functions in the elderly, including executive functions, processing speed, memory, and selective attention [18]. However, the effects of AET on WM performance were not clear in one meta-analysis [19], while another metaanalysis reported that intervention with various physical activities improved the WM performance using different WM outcomes [20]; thus, the findings were divided. Also, a recent study with the elderly investigated the effects of cognitive training involving several cognitive tasks, and physical training involving multiple physical activities including cardiovascular, and AET showed effects of cognitive training in one of the WM outcome measures, whereas physical training failed to show such effects [21]. Further, AET has been shown to increase prefrontal and temporal grey matter volume, anterior white matter volume, and neural activity of lateral frontal and parietal areas [22]. AET has also been reported to increase the efficiency of learning [22]. Part of this exercise effect may be mediated through increased secretion of brain-derived neurotrophic factor (BDNF), a key signal promoting activity-dependent neural plasticity [22].

The term dual-task activity refers to the performance of two different tasks simultaneously or in close succession. Dual-task activity is one of the functions of the central executive function system [23]. Also, simultaneously performed dual-task training that involves exercise has been shown to enhance cognitive executive functions, including general cognitive functions [24]. Dual-task training that involves overlapping tasks, usually two-alternative forced choice tasks that have to be performed simultaneously or in close succession, alters brain activity during cognitive tasks [25]. In particular, the effects of simultaneously performed dual-task training that combines a walking or standing activity (balance exercise) with cognitive training have been investigated [24]. However, the methods of these studies, including outcome measures, have varied. some studies recruited healthy older adults, whereas others recruited cognitively impaired adults [24]. Many of these studies, but not all, have found that this type of simultaneously performed dual-task training that involves physical activity leads to improved performance of global function measures such as the frontal assessment battery (FAB) [24]. Another recent meta-analysis also concluded that simultaneously combined physical and cognitive training is more successful than the use of physical training alone or cognitive training alone, though the improvement was somewhat confined to trained cognitive functions, rather than generalizing to other cognitive or daily living skills [26].

To summarize, we focused on SDAEWMT (combination of WMT, AET, and simultaneously performed dual-task training) because of the following reasons: (1) the development of an effective strategy to maintain cognitive abilities 
in older adults is important; (2) WMT, AET, and simultaneously performed dual-task training have all been shown to be effective for such purposes; and (3) AET leads to an increased secretion of BDNF, which facilitates neural plasticity. However, for older adults, this type of training (e.g., execution of walking and WM tasks simultaneously in real life) may be ecologically difficult because of the requirement of supervised exercise in combination with the implementation of effective computerized cognitive training tasks similar to previously developed systems [27]. In addition, previous studies have not investigated the effects of simultaneously performed dual-task training involving both AET and WMT (SDAEWMT) on neural systems.

To address these unresolved issues, we first designed AET incorporating the use of a recumbent ergocycle bike (Figure 1), which allows older subjects to sit in a stable position during training, thereby facilitating training that is both safe and ecologically applicable. Next, we designed a WMT program that used a portable console (3DS; Nintendo, Kyoto, Japan). Incorporating portable hardware (3DS) facilitated easy subject use during SDAEWMT. The combination of AET and WMT was adaptable to individual capacity and the physical condition of each older subject, enabling them to reach their optimal ecological level (for details, see Methods). We hypothesized that SDAEWMT is more effective than WMT or AET alone and/or (a) has effects on cognitive functions such as global cognitive functions, memory performance, attention, and executive functions, for which previous simultaneously performed dual-task training involving aerobic exercise or both WMT and AET have been effective, as described above; (b) alters brain structures and functional activities involving frontal and parietal areas where effects of WMT and AET have been observed, as described above, and that are critical for dual-task execution [25]; and (c) facilitates the improvement of WMT performance (greater improvement of training tasks' performance of WMT). The aims of the present study were to test these hypotheses and to investigate the effects of SDAEWMT on older adult cognitive functions, neural systems, and learning processes during cognitive training.

\section{Methods}

2.1. Subjects. For subject recruitment, study inclusion factors were the ability to walk without assistance, physician approval after assessment, right-handedness as determined using the Edinburgh Handedness Inventory (Oldfield, 1971), and age more than 65 years and less than 75 years. The right-handed subjects were recruited based on the custom of the MRI studies. Subjects were recruited from Sendai City. There were several exclusion criteria in this study and for details, see Supplemental Methods.

The Ethics Committee of Tohoku University approved all study procedures.

After an initial check of the inclusion and exclusion criteria, 102 right-handed older adults [65.9 \pm 13.7 (SD) years old] participated in a physician-administered health assessment involving an exercise electrocardiogram. After further exclusions and dropouts before and after random allocation

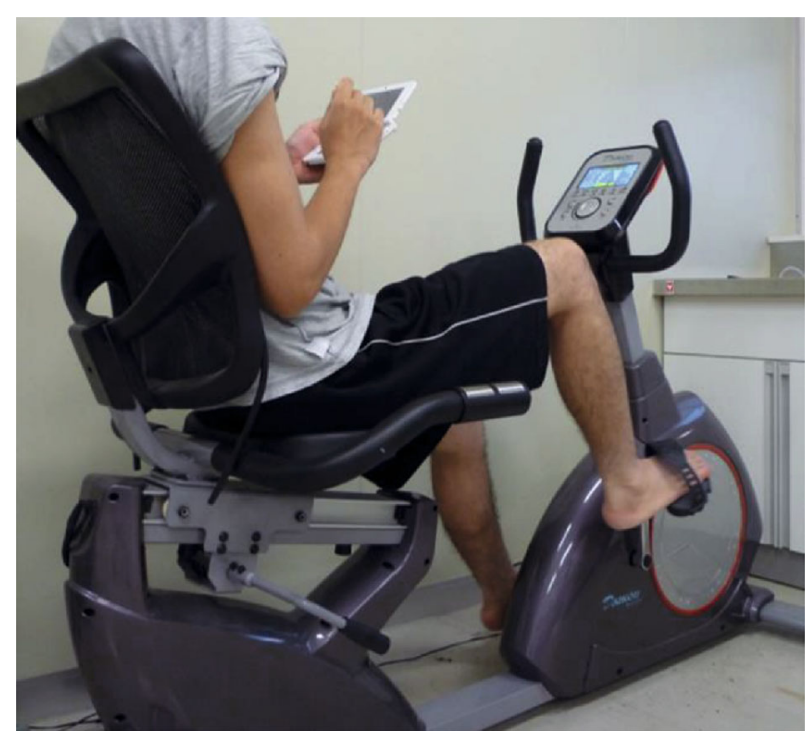

FIgURE 1: The SDAEWMT regimen conducted in the present study. Subjects were instructed to perform WMT implemented on a portable-type console (3DS) while riding a recumbent bike.

based on the random number generated through the excel file, a total of 93 subjects successfully completed the intervention (see Figure 2 for a detailed subject flowchart), with 30 subjects in the WMT group, 30 in the SDAEWMT group, and 33 in the AET group. Random allocation was performed using the Microsoft Excel program. Group age and sex ratios are presented in Table 1. No participant had a history of neurological or psychiatric illness according to a routine questionnaire that was created and is used in this laboratory. Handedness was evaluated using the Edinburgh Handedness Inventory [28]. Written informed consent was obtained from each individual for the interventions in which he/she participated. The three groups did not differ significantly in age, sex ratio, MMSE score, or FAB score $[P>0.1$ by analysis of variance (ANOVA)]. Subjects who misunderstood the rules for certain psychological measures were excluded from analyses involving those measures. Misunderstanding of the rules was judged according to "answers based on wrong rules" or "answers of chance level in the tasks with multiple choices".

2.2. Procedure. All the subjects were asked to engage in training three times each week in a laboratory located at the Institute of Development, Aging and Cancer, Tohoku University. If a subject was not available during a given week, he/she could make up for the lost sessions in the following weeks. Five subjects in the WMT group, 9 in the SDAEWMT group, and 8 in the AET group performed this procedure and attended four sessions in a week at least once. The first WMT and SDAEWMT group sessions were practice sessions to introduce the game software that would be utilized during training (see the next subsection for details). Each training session lasted for approximately $1 \mathrm{~h}$, and sessions were held over a period of 12 weeks. All the subjects underwent MRI and completed psychological evaluations immediately before and after this 12-week training period. 


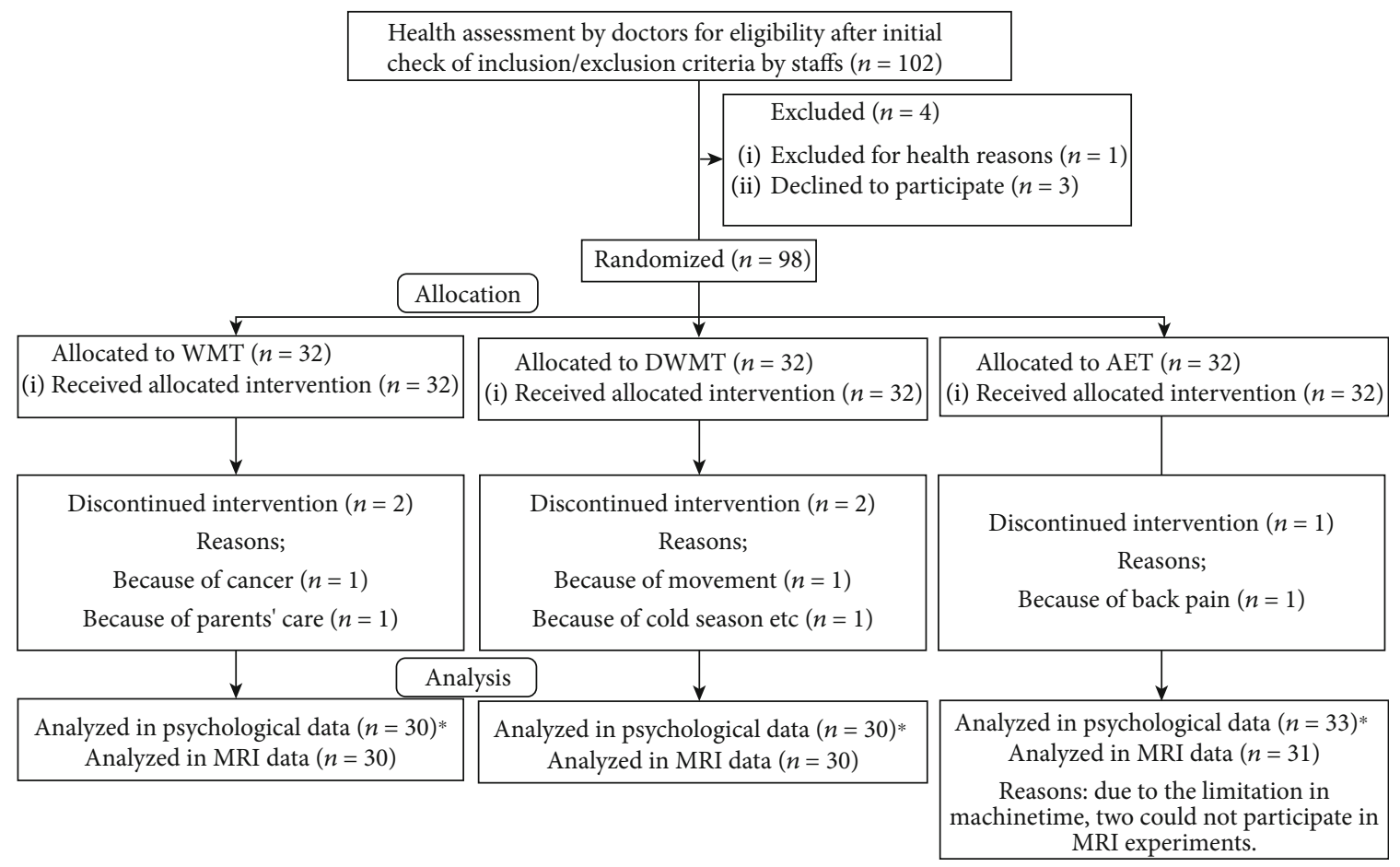

FIGURE 2: Study flowchart.

TABLE 1: Characteristics of subjects who successfully completed the assigned training sessions.

\begin{tabular}{|c|c|c|c|}
\hline & $\begin{array}{c}\text { WMT } \\
(N=30)\end{array}$ & $\begin{array}{c}\text { SDAEWMT } \\
(N=30)\end{array}$ & $\begin{array}{c}\text { AET } \\
(N=33)\end{array}$ \\
\hline Age, mean \pm SD & $68.77 \pm 2.94$ & $68.03 \pm 3.27$ & $69.3 \pm 3.37$ \\
\hline Female, $n$ & 19 & 18 & 21 \\
\hline MMSE-J, mean \pm SD & $28.27 \pm 1.39$ & $28.53 \pm 1.86$ & $28.00 \pm 1.81$ \\
\hline $\mathrm{VO}_{2} \max$ & $30.97 \pm 5.64$ & $30.53 \pm 6.73$ & $29.48 \pm 6.46$ \\
\hline Completed training sessions, mean \pm SD (range) & $\begin{array}{c}33.33 \pm 1.56 \\
(31-37)\end{array}$ & $\begin{array}{c}33.67 \pm 1.60 \\
(31-37)\end{array}$ & $\begin{array}{c}33.48 \pm 2.18 \\
\quad(27-37)\end{array}$ \\
\hline Education length* & $4.63 \pm 1.05$ & $4.90 \pm 1.16$ & $4.73 \pm 1.15$ \\
\hline
\end{tabular}

*For details of questions examining the education length of each participant, see our previous study's description of the questions that established the data on length of education of the participants' parents [1]. The same method was used.

2.3. Training Tasks. For WMT, we used the game suite "Brain Age: Concentration Training" published by Nintendo (Nintendo, Kyoto, Japan). This game includes eight WMT tasks, of which we used seven. Devilish reading, a reading span-like task, was excluded because it required the subject to read aloud, which may have disturbed other subjects playing in the same room. One session on an individual task ended when one trial ended after $5 \mathrm{~min}$ of training time (excluding interval times) had elapsed, after which the subjects proceeded to the next task. The order of the tasks was not specified, and the subjects could perform each task in an arbitrary order. For details of the training tasks, see Supplemental Methods. The schema of the tasks was presented in Figure 3.

The exact time taken for the subjects to complete the training session on each day was not measured. However, it takes at least 5 min to complete each task, and there are intervals between each trial during which the subjects could rest. Therefore, it usually took more than $45 \mathrm{~min}$ to complete the training session.

For AET, we used a recumbent ergocycle (DK-8718RP, Daikou, Japan) that electronically controls the workload. Using this ergocycle, the subjects could sit leaning back in a stable position during training. The training consisted of three sessions per week of $60 \mathrm{~min}$ each that included (i) 5 min of activity at low intensity so that all the subjects could achieve $35 \%-40 \%$ of their training intensity (see below for this calculation), (ii) $45 \mathrm{~min}$ of aerobic exercise on the ergocycle at moderate intensity based on the individual's capabilities and clinical status, and (iii) $10 \mathrm{~min}$ for cooling down and resting. Each subject's heart rate was monitored during all the training sessions by means of a wearable system which is 


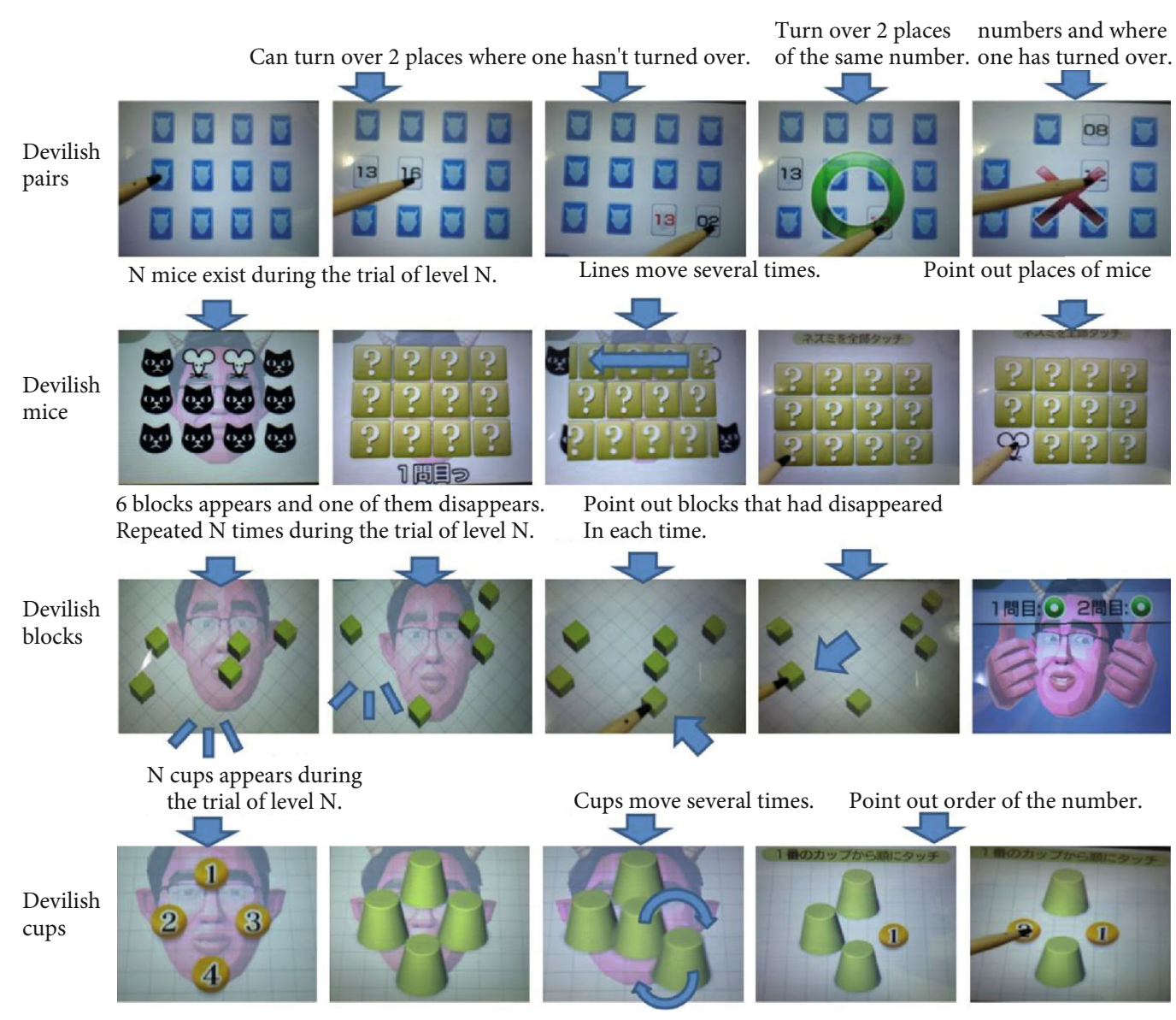

Figure 3: Schemata of four complex WMT tasks.

attached to the left hand and measures the heart rate as presented in the index finger. The target was between $40 \%$ and $50 \%$ of training intensity by Karovonen's formula [29] using the individual maximal heart rate estimated using Tanaka's formula [30]. The range was $40 \%$ to $50 \%$ training intensities (TI) which were calculated as follows.

(1) Estimation of one's own maximal heart rate (MHR). $\mathrm{MHR}=208-(.70 \times$ age in years $) \mathrm{bpm}[30]$

(2) Measurement of resting Heart Rate (RHR)

(3) Calculation of Heart Rate Reserve (HRR). HRR = MHR - RHR [29]

(1) Calculation of Heart rate. $40 \% \mathrm{TI}=\mathrm{HRR} \times 0.4+\mathrm{RHR}$ $.50 \% \mathrm{TI}=\mathrm{HPR} \times 0.5+\mathrm{RHR}[29]$

$40 \%$ to $50 \%$ TI may seem weak. However, we used $40 \%$ to $50 \% \mathrm{TI}$ in this study for the following reasons. First, prior to the experiment, we confirmed it was difficult to combine aerobic exercise of stronger TI with WMT in the setting of SDAEWMT, and this study is the study to investigate the effects of SDAEWMT. Second, as described above, previous simultaneously performed dual-task training paradigms involving exercise used walking (which is supposed to have weak TI) as an exercise to combine with cognitive training [24]. Third, a meta-analysis using data from 29 studies, involving 2049 participants, showed that there are no significant correlations between the TI of aerobic exercise on cognitive measures that showed the effects of AET (measures of attention and processing speed, executive function, and memory) and the effect size [19]; however, another metaanalysis that investigated the association between the status of aerobic exercise and cognitive functions in crosssectional studies showed that in cross-sectional studies, either an intensity of aerobic exercise that is too low or an intensity that is too high is not effective for cognitive functions [31]. Another meta-analysis, using the data from randomized controlled trial or quasi-experimental studies with appropriate comparison groups, targeted participants aged 65 years or above and who had been prescribed with low-intensity exercise in at least one study arm and reported that low-intensity exercise can improve both cognitive functions and affective state [32]. These previous studies demonstrate that our results cannot be dismissed due to the fact that our study used weaker TI [33]. The group difference of the 
preintervention aerobic capacity cannot explain the present findings, because this is a randomized controlled study and such differences should not occur and preintervention VO2max level did not differ among 3 groups (ANOVA, $P=0.630$, Table 1).

In SDAEWMT, the subjects were asked to perform the same cognitive training tasks as those described for WMT while performing the same aerobic exercises as those described for AET. If they did not finish WMT within 55 min of exercise, they were asked to keep cooling down while simultaneously completing WMT.

The length of one session of AET and SDAEWMT was determined from how long it takes to complete all the WMT tasks (about $45 \mathrm{~min}$ ) in the WMT group. Moreover, at least one meta-analysis of aerobic exercise showed that a training length of $30-45 \mathrm{~min} /$ session is more effective than training with longer sessions and training with shorter sessions [34].

Furthermore, after the experiment, a questionnaire with a 9-point Likert scale was administered to some participants to subjectively evaluate each training task (WMT: $N=21$, SDAEWMT: $N=23$, AET: $N=24$ ). There was no significant difference among the three groups in motivation toward the training tasks $[P=0.746, F=0.294$, WMT: $7.381 \pm 0.326$ (SEM), SDAEWMT: $7.304 \pm 0.312$ (SEM), AET: $7.625 \pm$ 0.305 (SEM)], fatigue of the subjects after the training $[P=0.618, \quad F=0.486, \quad$ WMT: $5.190 \pm 0.460 \quad$ (SEM), SDAEWMT: $5.435 \pm 0.439$ (SEM), AET: $4.833 \pm 0.430$ $(\mathrm{SEM})]$, satisfaction with the training tasks $[P=0.792, F=$ 0.234, WMT: $6.476 \pm 0.444$ (SEM), SDAEWMT: $6.261 \pm$ 0.424 (SEM), AET: $6.667 \pm 0.415$ (SEM)], or enjoyment during the training tasks $[P=0.402, F=0.925$, WMT: $7.143 \pm$ 0.348 (SEM), SDAEWMT: $6.870 \pm 0.332$ (SEM), AET: $7.500 \pm 0.325(\mathrm{SEM})]$. Therefore, these group differences do not seem to be substantial.

2.4. Psychological Outcome Measures. The following neuropsychological measures assessing specific cognitive domains were administered as pre- and posttraining evaluations. They do not include computerized tasks. All measures were administered to test the primary hypotheses of the present study (superiority of SDAEWMT over WMT and AET).

The details of tests (a), (b), (d), (f), (g), and (h) are provided in Supplemental Methods.

(a) Nonverbal reasoning. Nonverbal reasoning was assessed using Raven's advanced progressive matrix [35].

(b) Verbal WM. Verbal WM was tested by a digit span task.

(c) Immediate recall. Logical memory I (LM), which involves immediate recall after hearing a story, was evaluated using a subtest of the Wechsler Memory Scale-Revised (WMS-R) [36]. The test material consisted of two short paragraph-length stories (story A and story B). In the LM task, the subjects were required to memorize short story A, and soon after that, subjects were told to tell out loud the story they remembered. Then, the same procedure was repeated for story B. Performance was scored by the number of story units recalled as specified in the WMS-R scoring pro- tocol. The primary measure for this task was the sum of the number of correct story units.

(d) Attention. Attention was measured by the digit cancellation task (D-CAT) [37]. For this measure, we used the mean number of successfully cancelled items from the three trials.

(e) Frontal lobe and executive function. FAB was employed for this evaluation [38]. The following description of FAB is a summary from our previous study [39]. FAB consists of six subtests, namely, those for similarities (conceptualization), lexical fluency (mental flexibility), motor series (programming), conflicting instructions (sensitivity to interference), go-no go (inhibitory control), and prehension behavior (environmental autonomy). FAB is scored from 0 to 18. Lower scores of FAB indicate greater degrees of executive dysfunction. The primary measure is the total score of this task $(\max =18)$. The full details of these subtests have been described extensively in a previous study [38].

(f) Processing speed. Processing speed was evaluated by the Symbol Search [40] and Digit Symbol tasks [40].

(g) Creativity measured by divergent thinking. The SA creativity test was employed to evaluate creativity [41-43]. This task involves three subtasks: (i) generate unique ways of using typical objects, (ii) imagine desirable functions for ordinary objects, and (iii) imagine the consequences of unimaginable things happening. The SA test scores the four dimensions of the creative process (fluency, originality, elaboration, and flexibility). In this study, the sum of the graded scores of the four dimensions was used for analysis.

(h) General cognitive functions. MMSE [44], the screening instrument most widely used for detecting cognitive impairment in older adults, was used to evaluate general cognition. Because of an apparent ceiling effect, this task was not used for the assessment of training effects.

(i) Inhibition. The Stroop task (Hakoda's version) [45] was used to evaluate response inhibition and impulsivity. However, results were not analyzed because a substantial proportion of the subjects could not see yellow. Subjects were elderly, and with aging, sometimes the lenses of their eyes had become more yellow, making it difficult for such subjects to differentiate yellow from white (which is the background color of the test). In addition, we could not provide instructions to testers on how to address this problem; therefore, different testers provided different instructions to the subjects.

Because of misunderstanding of the rules, one subject in the WMT group was excluded from the analysis of the LM task. Two subjects in the WMT group were excluded from the analysis of the Symbol Search task. One subject in the WMT group, one subject in the SDAEWMT group, and three subjects in the AET group were excluded from the analysis of D-CAT.

Several questionnaires designed to assess the traits or states of the subjects were collected but are not described here. Physical tasks involving walking and dual tasks were also performed, but these results will be reported by other authors.

The preassessment of psychological outcome measures was conducted in a double-blind manner. We used exactly the same versions of the test for the pre- and postmeasures, 


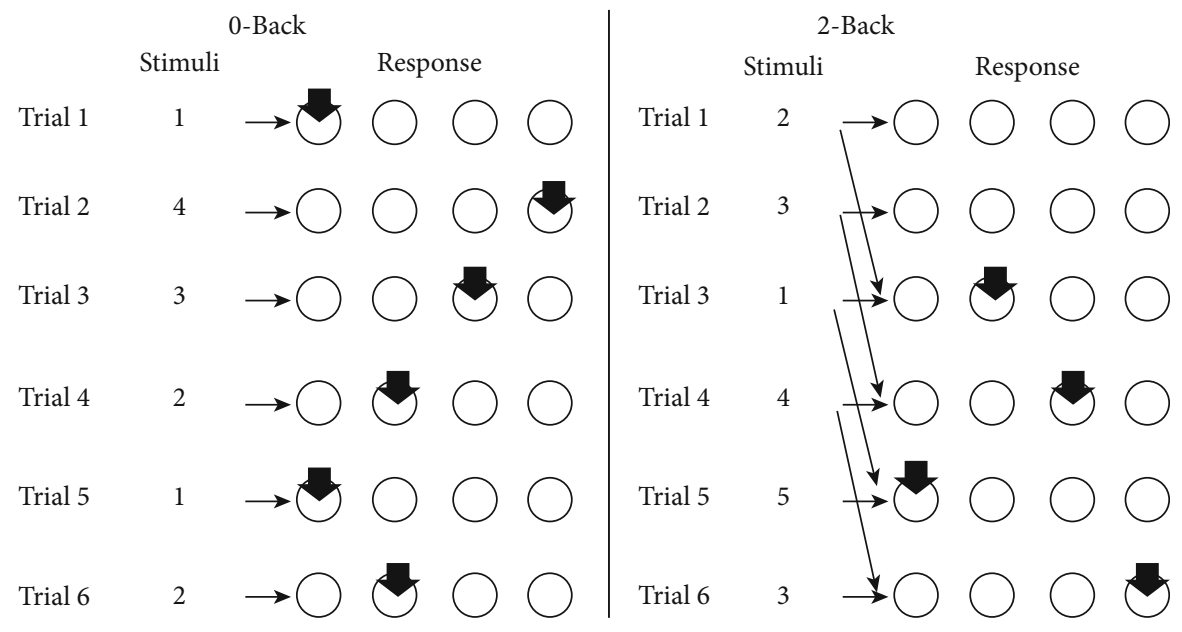

FIGURE 4: Illustration of the N-back task, showing six trials of the 0-back level (left) and six trials of the 2-back level (right), together with the appropriate responses. At the 0 -back level, subjects only have to push a button spatially corresponding to the location of a number on the screen. At the 2-back level, subjects must push a button spatially corresponding to a number that appeared two trials earlier.

except for the S-A creativity test, which had different versions for pre and post [41].

2.5. Functional Magnetic Resonance Imaging (fMRI) Tasks. fMRI was used to map training-induced changes in brain activity related to WM. An $N$-back task commonly used for fMRI studies was used to detect training-related changes in brain activity patterns related to these cognitive processes. In this version using digits as stimuli, there were two conditions: 0-back for testing simple cognitive processes and 2back for testing WM. These are described in our previous publications $[46,47]$. In brief, the subjects performed an $N$ -back task using four numbers (i.e., 1-4). The subjects were asked to memorize a series of numbers and their temporal order, update the list of recent items, and select the responses that corresponded to the previously observed stimuli according to the $N$-back rule. The stimuli were shown in a random sequence on the screen. Each stimulus was presented for $0.5 \mathrm{~s}$. Following this, a fixation symbol was presented for $2.5 \mathrm{~s}$, and the next stimulus was then presented. The subjects had to respond either directly following the stimulus (0-back) or after a delay of two stimulus presentations (2-back). The schema of the task is presented in Figure 4. The task level of the memory load was displayed above the stimuli starting $2 \mathrm{~s}$ before task onset and remained visible during the task period. A single-condition block consisted of a 2-s presentation of the task instructions (e.g., "2-back"), followed by the 18-s actual task period. There were five blocks for each period. Because there are various strategies for performing $\mathrm{N}$-back tasks, the subjects were requested to follow a specific strategy as instructed. The subjects were told to update memory $N$ by $N$ (e.g., in the 2-back task, they were asked to update their memory two by two) and not one by one. For instance, in the 2-back task, they first had to remember two numbers presented, and then, while they pushed the corresponding two buttons, they were asked to remember the two incoming numbers.

Visual stimuli were presented using Presentation Software (Neurobehavioral Systems, Inc., Albany, CA, USA).
Stimuli were projected to the subjects via a screen positioned at the head-end of the board. The subjects viewed the screen through a mirror attached to the head coil. A fiber-optic light-sensitive key press button box was used to record the subjects' behavior. In contrast to other variants of the $N$ -back task, only accuracy was used as a measure of performance in this study. The reaction time did not reflect performance because in the 2-back task, prior to the presentation of the next stimuli, subjects already knew which button they should push (as determined by the preceding stimuli) after the presentation of the following stimuli and the timing of the presentation of the next stimuli before they were allowed to respond (see Figure 4).

In this study, our focus was on the frontal and parietal areas, and activation in these regions occurs regardless whether the task is 2-back or 0-back in mostly similar areas, although the magnitude may differ. Furthermore, differences in brain activity between patients with schizophrenia and control subjects were mostly similar, regardless of whether the task was a 0-back task or 2-back task, especially in these areas (i.e., subtracting the activity during the 0-back task from the brain activity during the 2-back task substantially eliminates the group differences) $[48,49]$.

2.6. Image Acquisition and Analysis. MRI data were acquired using a $3 \mathrm{~T}$ Philips Intera Achieva scanner. Diffusionweighted data were acquired using a spin-echo EPI sequence [repetition time $(\mathrm{TR})=10293 \mathrm{~ms}$, echo time $(\mathrm{TE})=55 \mathrm{~ms}$, field of view $(\mathrm{FOV})=22.4 \mathrm{~cm}, 2 \times 2 \times 2 \mathrm{~mm} 3$ voxels, 60 slices, SENSE reduction factor $=2$, number of acquisitions $=$ $1]$. The diffusion weighting was isotropically distributed along 32 directions ( $b$ value $=1,000 \mathrm{~s} / \mathrm{mm} 2$ ). In addition, three images with no diffusion weighting ( $b$ value $=0 \mathrm{~s} / \mathrm{mm} 2) \quad(b=0$ images $)$ were acquired using a spin-echo EPI sequence $(\mathrm{TR}=10293 \mathrm{~ms}, \mathrm{TE}=55 \mathrm{~ms}$, FOV $=22.4 \mathrm{~cm}, 2 \times 2 \times 2 \mathrm{~mm} 3$ voxels, 60 slices). From the collected images, fractional anisotropy (FA) and mean diffusivity (MD) maps were calculated. Forty-two transaxial gradient-echo images $\left(\mathrm{TE}=30 \mathrm{~ms}\right.$, flip angle $=90^{\circ}$, slice 
thickness $=3 \mathrm{~mm}$, FOV $=192 \mathrm{~mm}$, matrix $=64 \times 64)$ covering the entire brain were acquired at TR of $2.5 \mathrm{~s}$ using an echo planar sequence. For the $N$-back session, 135 functional volumes were obtained.

2.7. Preprocessing of Diffusion Data. The MD and FA measures of diffusion tensor imaging (DTI) [50] can detect unique aspects of brain microstructural neural plasticity $[15,51]$, including those caused by WMT $[15,52,53]$. A reduction in $\mathrm{MD}$ reflects an increase in the tissue density of various cellular structures, including capillaries, synapses, spines, and macromolecular proteins, as well as changes in the properties of myelin, membranes, and axons; the shapes of neurons or glia; and tissue organization. However, MD is not specific to any one of these microlevel changes [50,51]. On the other hand, an increase in FA is thought to have a relatively greater association with changes in microstructural properties related to brain connectivity, including myelination, axonal membrane thickness and diameter, and degree of axonal parallel organization [50, 52].

Preprocessing of imaging data was performed using Parametric Mapping software (SPM8; Wellcome Department of Cognitive Neurology, London, UK) implemented in Matlab (Mathworks Inc., Natick, MA, USA). The descriptions in this subsection are a summary from our previous publication [15]. Pre- and posttraining MD and FA maps were independently segmented and normalized using the previously described diffeomorphic anatomical registration through an exponentiated Lie algebra (DARTEL)-based method (Takeuchi et al., 2013a). This method accounts not only for the outer edge but also for the signal distribution within the white matter during registration. The reason for the independent normalization has been described previously (Takeuchi et al., 2013a).

From the pre- and postintervention normalized images of the normalized MD map, areas not strongly likely to be gray or white matter in our custom template (defined by "gray matter tissue probability + white matter tissue probability < 0.99 ") were removed. Then, from the pre- and postintervention normalized images of normalized FA map, areas not strongly likely to be white matter in our custom template (defined by "white matter tissue probability < 0.99 ") were removed. Subsequently, normalized MD and FA images were smoothed by convolution with an isotropic Gaussian kernel of 10 - and $8-\mathrm{mm}$ full-width at half maximum, respectively. These images were used for the group analysis described below.

2.8. Preprocessing and Data Analysis for Functional Activation Data. Preprocessing and data analysis were performed using SPM8 implemented in Matlab. Prior to analysis, BOLD images from the pretraining scan and the posttraining scan were realigned and resliced to the mean BOLD image of all pretraining scans. The images were then corrected for slice timing, coregistered to a $b=0$ diffusionweighted image, and spatially normalized using the parameter of the abovementioned normalization of FA images to form images with $3 \times 3 \times 3 \mathrm{~mm} 3$ voxels.
A design matrix was fitted to each subject with one regressor for each task condition (0-back and 2-back in the $N$-back task) using the standard hemodynamic response function (HRF). The design matrix weighted each raw image according to its overall variability to reduce the impact of movement artifacts [54]. After estimation, beta images were smoothed (8-mm full-width at half maximum) and either taken to the second level or subjected to random-effect analysis. We removed low-frequency fluctuations using a highpass filter with a cut-off value of $128 \mathrm{~s}$. Individual-level statistical analyses were performed using a general linear model.

In individual analyses, we focused on the activation change and compared activation relative to the conditions (0-/2-back versus rest) before and after an intervention period. The resulting maps for each subject represented changes between the pre- and postmeasures of brain activity during the corresponding conditions as well as the brain activity during the corresponding conditions in the premeasures, which were then included in a group analysis.

2.9. Preprocessing of Structural Data. Preprocessing of imaging data was performed using SPM12 implemented in Matlab. Using the Pairwise Longitudinal Registration toolbox [55], the midpoint average template images were generated for each participant as well as maps of differences between the Jacobian determinants and of pre- and postintervention T1-weighted structural images using default parameter settings. The midpoint average image of each subject was then segmented, and the Thorough Clean option was used to remove any odd voxels. Affine regularization was performed in accordance with the East Asian brain with a sampling distance (the approximate distance between the sampled points when estimating the model parameters) of $1 \mathrm{~mm}$. Default parameter settings were used for other parameters in the segmentation process. Then, by multiplying maps of differences between the Jacobian determinants of pre- and postintervention images and gray matter segments of the midpoint average image of each subject, images of gray matter volume change were generated. Then, diffeomorphic anatomical registration was performed using DARTEL processes. Here, we used imported DARTEL images of gray matter and white matter for the processes created above. The DARTEL template was created from the entire set of subjects in this experiment, and the default parameter settings were used for the DARTEL process. Then, using the parameters generated for each participant, the images of gray matter change were normalized and smoothed by convolving each with an isotropic Gaussian kernel of $8 \mathrm{~mm}$ FWHM.

2.10. Statistical Group-Level Analysis of Imaging and Behavioral Data. There were numerous potential contrasts in this study, because there were three groups with two factors (aerobic exercise and WMT). However, we only tested two-step analyses to confirm our hypothesis. The first step was the superiority of SDAEWMT over single training (i.e., comparison of SDAEWMT with the combined groups of WMT and AET) which was followed by the second step that confirmed the superiority of SDAEWMT over AET as well as the superiority of SDAEWMT over WMT (the comparison 
between SDAEWMT and AET and the comparison between SDAEWMT and WMT). Behavioral data were analyzed using SPSS 22.0 (SPSS Inc., Chicago, IL). In behavioral analysis, because the superiority of SDAEWMT was the primary study hypothesis (and we are not aware of any study indicating that dual-task training is inferior to single-task training in terms of cognitive transfer), test-retest changes within the SDAEWMT group were compared with those in the WMT or AET group using one-tailed, one-way analysis of covariance (ANCOVA) with the posttest measures as dependent variables and pretest scores as independent variables $(P<0.05)$. This ANCOVA design was selected to increase the sensitivity of analysis. As described in our previous study [56], we employed ANCOVAs instead of repeated measure ANOVAs to control the effects of pretest scores. Statistical experts strongly recommend to use ANCOVA instead of repeated measure ANOVA in this type of study design [57]. With randomized designs, the purpose of ANCOVA is to reduce error variance, whereas with nonrandomized designs (or with analyses involving substantial preexisting group differences), ANCOVA is used to adjust the posttest means for pretest differences among groups [57]. One might recommend that posttest scores be used, instead of using the differences between pre- and posttest measures. However, in practice, when the pretest scores are included as covariates, the two analyses return the same $P$ value.

The use of a one-tailed test was consistent with previous studies $[27,47,58,59]$. In tests of the effects of SDAEWMT group compared with those of others, for post hoc comparisons between SDAEWMT and AET groups as well as comparisons between SDAEWMT and WMT groups, when ANCOVAs showed the same pattern $(P<0.1)$ of superiority for the effects of SDAEWMT, the results were regarded as showing the superiority of SDAEWMT over single training (here the main contrast is SDAEWMT vs AET + WMT; however, the existence of similar trends in the contrasts of (SDAEWMT vs AET) and (SDAEWMT vs WMT) was confirmed with a more liberal threshold). In all nonwhole-brain analyses (i.e., behavioral analyses) performed in this study, results with a threshold of $P<0.05$ corrected for false discovery rate (FDR) using the graphically sharpened method [60] were considered statistically significant. The correction for multiple comparisons using this method was applied to the results of 10 ANCOVAs that tested the superiority of SDAEWMT over single training. ANOVAs were utilized to evaluate group differences in cognitive performance before the intervention. In group-level imaging analyses, we tested for group-wise differences in the change of each measure across the entire brain.

For determining the MD, FA, and brain activity, analyses were performed using Biological Parametrical Mapping [61], the extension of SPM, which allow the inclusion of signal of other images at every voxel, to perform voxel-by-voxel ANCOVAs. In analyses of MD and FA, we performed voxel-wise ANCOVAs with the values of postscans at each voxel as dependent variables and the values for the prescans at each voxel as independent variables. In analysis of the $N$ -back task, we performed voxel-wise ANCOVAs with the contrast of activity (0-back or 2-back) changes between pre- and postscans at each voxel as dependent variables and the corresponding value for the prescan at each voxel, the accuracy of the corresponding tasks in the prescan, and its preto postchanges (post-pre) as independent variables. For the $\mathrm{N}$-back task, only the subjects who achieved an accuracy of $80 \%$ or higher during both pre- and postsessions were included in the analysis, because when the task difficulty was too high for the subjects, brain activity showed nonlinear patterns and such nonlinear changes could not be assessed by the statistical tests applied [62]. In other words, areas that showed an increase in activity as the load increased started to exhibit a decrease in activity when the task difficulty became too high. By this inclusion, we could focus only on linear changes and could ignore the effects of "not doing the tasks properly." Consistently, Jansma et al. [48] showed that $75 \%$ accuracy is too low for this type of $N$-back task among healthy participants to detect linear changes (brain activity in relevant areas increased from 0-back to 2-back task in which accuracy was about $82 \%$, but dropped substantially in the 3-back condition in which the accuracy was about $72.5 \%$ in the case of healthy participants). Since the random response can achieve 25\% accuracy rate in this task, $75 \%$ accuracy is actually not high, and it means they are substantially failing. Consequently, 13 WMT group subjects (mean accuracy, pre: $90.0 \pm 6.8 \%$, post: $91.9 \pm 7.2 \%), \quad 12$ SDWMAET group subjects (mean accuracy, pre: $90.8 \pm 8.1$ $\%$, post: $94.2 \pm 5.3$ ), and 13 AET group subjects (mean accuracy, pre: $89.6 \pm 6.6 \%$, post: $91.5 \pm 6.0 \%$ ) were included in analyses of the 2-back task. Without surprisingly, there were no significant differences of pre- to postchanges in accuracy among these subjects after correcting preintervention accuracy. 28 WMT group subjects, 26 SDWMAET group subjects, and 27 AET group subjects were included in analyses of the 0 -back task. In behavioral analyses of the $N$-back task, only the subjects who performed the task without misunderstanding of the rules were included from analyses. In these neuroimaging analyses, the effects of SDAEWMT compared with those of single training were tested using the contrasts of $[-1 \times$ WMT $2 \times$ SDAEWMT $-1 \times \mathrm{AET}]$ or $[1 \times \mathrm{WMT}-2 \times$ SDAEWMT $1 \times$ AET $]$ with the uncorrected masks of $[-1 \times$ WMT $1 \times$ SDAEWMT $]$ or $[1 \times$ WMT $-1 \times$ SDAEWMT $]$ and $[1 \times$ SDAEWMT $-1 \times$ AET $]$ or $[-1 \times$ SDAEWMT $1 \times$ AET] $(P<0.05$, uncorrected). Regions with significance were inferred using cluster-level statistics [63]. Only clusters with $P<.05$, after correction for multiple comparisons at cluster size, with a voxel-level cluster-determining threshold of $P<$ .001 (uncorrected) were considered statistically significant.

In the group-level imaging analysis of rGMV, we tested for group-wise differences in the change in rGMV across the whole brain with the same contrasts. We used the oneway ANOVA option in SPM8. In the imaging analysis, the effects of training with TCSSL were estimated by comparing differences between the groups of rGMV, as calculated above at each voxel. This analysis was limited to areas where the average rGMV value for the segmented and normalized mean images of pre- and postscans of all participants was greater than 0.1. A multiple comparison correction was performed using threshold-free cluster enhancement (TFCE) [64] with randomized (5,000 permutations) nonparametric 
TABLE 2: Highest performance scores (mean $\pm \mathrm{SD}$ ) of WMT and SDAEWMT groups on trained WM tasks among the second to fourth training sessions and final three training sessions ${ }^{\mathrm{a}}$.

\begin{tabular}{lcccc}
\hline & \multicolumn{2}{c}{ WMT } & \multicolumn{2}{c}{ SDAEWMT } \\
& First three sessions & Last three sessions & First three sessions & Last three sessions \\
\hline Devilish calculations & $2.08 \pm 0.23$ & $3.05 \pm 0.39$ & $2.02 \pm 0.24$ & $2.98 \pm 0.42$ \\
Devilish pairs (oni-mekuri) & $5.93 \pm 0.93$ & $7.63 \pm 1.25$ & $6.13 \pm 0.92$ & $7.57 \pm 1.45$ \\
Devilish mice & $2.47 \pm 0.73$ & $3.15 \pm 0.79$ & $2.38 \pm 0.40$ & $3.18 \pm 0.65$ \\
Devilish shapes & $2.02 \pm 0.09$ & $2.15 \pm 0.39$ & $2 \pm 0$ & $2.13 \pm 0.29$ \\
Devilish blocks & $4.43 \pm 1.05$ & $5.93 \pm 1.31$ & $4.43 \pm 1.12$ & $5.70 \pm 1.16$ \\
Devilish cups & $4.68 \pm 0.56$ & $5.62 \pm 0.80$ & $4.62 \pm 0.56$ & $5.62 \pm 0.8$ \\
Devilish listening & $1.75 \pm 0.34$ & $2.30 \pm 0.40$ & $1.57 \pm 0.40$ & $2.27 \pm 0.7$ \\
\hline
\end{tabular}

${ }^{a}$ The data from the first training session were excluded because the first training session mainly consisted of practice and explanation of the training tasks.

testing using the TFCE toolbox (http://dbm.neuro.uni-jena .de/tfce/). We applied a threshold of family-wise error corrected at $P<0.05$. The standard cluster size test is inappropriate in voxel-based morphometry [65], whereas a permutationbased test is assumed to be correct. However, the abovementioned analysis using BPM cannot be applied to rGMV analysis because the TFCE toolbox cannot be used in BPM, which is why we chose this statistical design for rGMV analysis.

\section{Results}

3.1. Basic Data and Results of Training Data. The numbers of training sessions for subjects who completed the intervention in each training group are presented in Table 1 . There were no significant preexisting group differences in the general cognitive function (MMSE score, $\mathrm{FAB}$ ), $\mathrm{VO}_{2} \mathrm{Max}$, education length, working memory, or short-term memory performance (digit span performance, 2-back task performance), as shown in Table 1 (ANOVA, $P>0.05$ ).

Training data for WMT and SDAEWMT are presented in Table 2. The increase in the average of the highest performance scores $(N)$ for trained WM tasks from the first three training sessions (excluding the first session, which was completed as a practice and explanation session) to the last three sessions was not significantly greater with SDAEWMT than with WMT after correcting for the highest performances of trained WM tasks during the first three training sessions, excluding the first session $(P=0.833$ by one-tailed ANCOVA). The best performance among the three sessions was used because the brevity of each session for each training task would lead to instability of results if we used the data from just one training day. This same group procedure was applied previously $[52,56,66]$.

Overall, the expected improvement of cognitive training task performance was not observed. On the other hand, most subjects successfully completed DAEMT training as the performance of each specific training task was comparable with that of WMT group subjects.

We also recorded subjects' heart rates during the resting state prior to exercise (HRRSBE) in each training session and used as one measure of cardiovascular function. The mean \pm SD lowest HRRSBE from the first three training sessions (excluding the first session, which was completed as a practice and explanation session) and the last three training sessions were $75.61 \pm 8.37$ beats per minute $(\mathrm{bpm})$ and $70.09 \pm 8.48 \mathrm{bpm}$, respectively, in the AET group, and 76.63 $\pm 10.46 \mathrm{bpm}$ and $74.07 \pm 6.59 \mathrm{bpm}$, respectively, in the SDAEWMT group (we focused on the best performance among the three training days to prevent instability of the results based on a single trial. This method is consistent with those of our previous studies $[15,52,56])$. The decrease in HRRSBE in the AET group tended to be greater than that in the SDAEWMT group after correcting for the effect of the lowest HPRSBE value recorded during the first three training sessions, excluding the first session $(P=0.052$, twotailed ANCOVA). This finding suggests that although all the subjects in AET and SDAEWMT groups received the same instructions to keep their heart rate within a fixed range, the subjects in the SDAEWMT group may have been unable to follow this instruction all the time when they also had to concentrate on cognitive training. Therefore, the effects of aerobic exercise on HRRSBE may have been reduced during SDAEWMT.

3.2. Analyses of Cognitive Test Data and fMRI Task Behavioral Data. Compared with the WMT or AET group, the SDAEWMT group showed a significantly greater increase in 2-back task accuracy and FAB score (for statistical values including raw $P$ values, see Table 3 ). This difference remained significant after correcting for multiple comparisons (both $P=0.040$, corrected for FDR). However, the SDAEWMT group did not show a significantly larger improvement in 2-back task accuracy than the WMT group. The greater increase in FAB performance in the SDAEWMT group than in the WMT or AET group was moderated by the greater increase in the SDAEWMT group than in the WMT group as well as the significantly greater increase in the SDAEWMT group than in the AET group (Table 3).

3.3. MD Analyses. We also compared MD changes in the SDAEWMT group with those in the WMT and AET groups. This analysis revealed a significant SDAEWMT-related decrease from pre- to postmeasurement in MD regions, including areas adjacent to the right dorsolateral prefrontal cortex (DLPFC) and dorsal anterior cingulate cortex, areas in the right orbitofrontal cortex, areas in the left 
TABLe 3: Pre- and posttest scores for psychological measures (mean \pm SEM).

\begin{tabular}{lcccccccc}
\hline & \multicolumn{2}{c}{ WMT } & \multicolumn{2}{c}{ SDAEWMT } & AET & $\begin{array}{c}\text { Raw } P \text { value }{ }^{\mathrm{a}} \\
\text { SDAEWMT } \\
\text { vs. others }\end{array}$ & $\begin{array}{c}P \text { value }^{\mathrm{b}} \\
\text { Pretest } \\
\text { differences }\end{array}$ \\
\hline 0-back accuracy & $95.06 \pm 7.09$ & $96.89 \pm 4.38$ & $93.10 \pm 7.88$ & $96.21 \pm 8.01$ & $94.22 \pm 8.82$ & $93.87 \pm 9.77$ & 0.260 & 0.656 \\
2-back accuracy & $73.52 \pm 20.81$ & $88.52 \pm 11.45$ & $73.52 \pm 20.9$ & $90.00 \pm 9.81$ & $74.68 \pm 20.16$ & $76.45 \pm 20.53$ & $0.008^{\mathrm{c}}$ & 0.970 \\
RAPM & $12.97 \pm 4.45$ & $13.97 \pm 5.02$ & $14.67 \pm 5.41$ & $15.50 \pm 5.07$ & $13.67 \pm 4.50$ & $13.39 \pm 5.67$ & 0.179 & 0.401 \\
Digit span & $14.20 \pm 3.05$ & $15.00 \pm 2.76$ & $13.90 \pm 2.70$ & $14.87 \pm 3.03$ & $12.76 \pm 2.87$ & $14.52 \pm 3.19$ & 0.664 & 0.121 \\
Logical memory & $22.41 \pm 6.12$ & $26.69 \pm 6.61$ & $25.37 \pm 6.85$ & $26.07 \pm 6.5$ & $21.39 \pm 6.58$ & $23.67 \pm 7.87$ & 0.938 & 0.056 \\
D-CAT & $41.63 \pm 4.98$ & $41.95 \pm 6.68$ & $42.33 \pm 6.93$ & $42.83 \pm 6.53$ & $41.59 \pm 6.85$ & $42.21 \pm 7.05$ & 0.449 & 0.884 \\
FAB & $15.57 \pm 1.65$ & $15.07 \pm 1.9$ & $15.17 \pm 2.1$ & $15.6 \pm 1.70$ & $15.03 \pm 1.82$ & $14.21 \pm 2.04$ & $0.008^{\mathrm{d}}$ & 0.514 \\
Symbol search & $34.00 \pm 5.21$ & $35.46 \pm 5.55$ & $34.07 \pm 5.14$ & $34.1 \pm 5.44$ & $32.15 \pm 5.00$ & $34.36 \pm 6.21$ & 0.975 & 0.257 \\
Digit symbol & $66.83 \pm 14.39$ & $67.73 \pm 12.3$ & $65.90 \pm 11.46$ & $69.63 \pm 12.51$ & $63.45 \pm 13.26$ & $69.48 \pm 14.23$ & 0.430 & 0.581 \\
SACT & $17.67 \pm 5.15$ & $18.80 \pm 6.08$ & $15.83 \pm 4.79$ & $16.83 \pm 4.82$ & $15.30 \pm 5.57$ & $16.83 \pm 4.82$ & 0.837 & 0.187 \\
\hline
\end{tabular}

${ }^{a}$ One-way analysis of covariance (ANCOVA) tests comparing the SDAEWMT group with the other two groups for test-retest differences, with psychological measures as dependent variables and pretest scores for the psychological measures as covariates. For all tests, one-tailed tests were performed to assess the superiority of SDAEWMT. ' One-way ANOVAs comparing the pretest scores of the three groups. 'One-tailed, one-way ANCOVAs revealed that the increase in the SDAEWMT group was not significantly greater than that in the WMT group $(P=0.302)$ but that there was a significantly greater increase in the SDAEWMT group than in the AET group $(P=0.0003) .{ }^{\mathrm{d}}$ One-tailed, one-way ANCOVAs revealed a trend toward a greater increase in the SDAEWMT group than in the WMT group $(P=0.096)$ and a significantly greater increase in the SDAEWMT group than in the AET group $(P=0.003)$.

hippocampus, midbrain areas involving the bilateral substantia nigra, ventral tegmental area, periaqueductal gray and reticular formation, right ventral anterior cingulate cortex, and part of the left putamen and left pallidum (Figure 5(a), Table 4). There were no significant results of SDAEWMTrelated increase of MD from before measurement to after measurement.

3.4. FA Analyses. When FA for the SDAEWMT group was compared with that for the WMT and AET groups, there were no significant results of SDAEWMT-related changes of FA from before measurement to after measurement.

3.5. Brain Activity Analyses. Brain activity was assessed during the 2-back task, and the difference between the pre- and posttraining measurements was compared among the groups. The SDAEWMT group showed a significant increase in brain activity (meaning that the brain activity corresponding to the contrast of post versus pre is greater than in other groups) in the left temporoparietal junction [MNI coordinates: $x, y, z=-63,-42,15 ; t=4.68 ; P=0.002\left(3159 \mathrm{~mm}^{3}\right)$, corrected for multiple comparisons with the cluster determining threshold of $P<0.001]$ and in anatomical clusters that included the right temporoparietal junction [MNI coordinates: $x, y, z=69,-36,27 ; t=4.68 ; P=0.007\left(2430 \mathrm{~mm}^{3}\right)$, corrected for multiple comparisons with the cluster determining threshold of $P<0.001$ ] and the right superior temporal gyrus compared with the WMT and AET groups (Figure 6(a)). There were no significant results of SDAEWMT-related decrease of brain activity from before measurement to after measurement.

3.6. $r G M V$ Analyses. The SDAEWMT-related changes in the rGMV from before measurement to after measurement were not statistically significant.

\section{Discussion}

In the present study, we developed and successfully implemented an ecologically applicable SDAEWMT (simultaneously performed dual-task training involving both aerobic exercise training and working memory training) method and examined its effects on cognitive function (subjects could successfully perform cognitive training while doing the exercise in this setting), brain structure, and brain activity patterns in comparison with those of WMT and AET methods. We showed that (a) SDAEWMT significantly improved the performance of the FAB, but there were no significant results in other broader transfer measures. These results were only partly consistent with our hypotheses that (a) SDAEWMT would be more effective than WMT or AET alone and/or that SDAEWMT would have effects on cognitive functions such as global cognitive functions, memory performance, attention, and executive functions, and (b) SDAEWMT resulted in decreases in the MD in the frontal and subcortical brain areas involving the dopaminergic system. And SDAEWMT produced an increase in WM-related brain activity in the bilateral temporoparietal junction. These results were partly overlapping and partly distinctive compared to our hypothesis (b), which SDAEWMT would alter brain structures and functional activities involving frontal and parietal areas. (c) SDAEWMT did not result in a greater improvement of the performance of WMT tasks compared with WMT alone, which is inconsistent with our hypothesis that SDAEWMT facilitates the improvement of WMT performance.

The present behavioral results suggest that SDAEWMT has a specific effect on executive function; however, there was no evidence of broader transfer effects or enhanced learning ability. This result was based on results showing that SDAEWMT significantly improved the performance of FAB, 

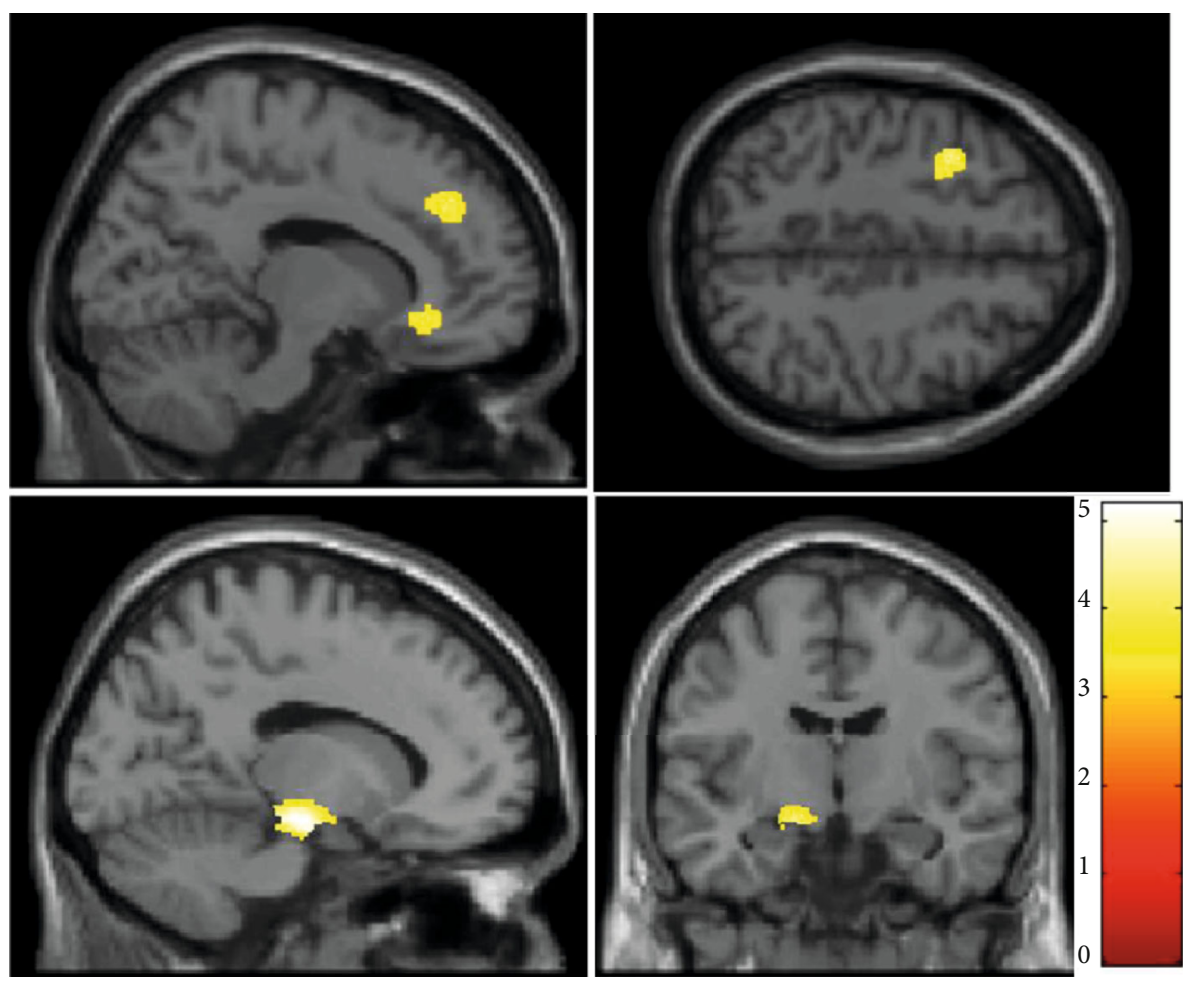

Figure 5: Change in diffusion imaging measures related to SDAEWMT. Decrease in mean diffusivity (MD) in the SDAEWMT group compared with that in the WMT and AET groups. The results are shown with a threshold of $P<0.05$, corrected for multiple comparisons at the cluster level, with an underlying voxel-level $P<0.001$, uncorrected. Compared with the WMT and AET groups, SDAEWMT training resulted in decreased MD in areas adjacent to the right dorsolateral prefrontal cortex (DLPFC) and dorsal anterior cingulate cortex (dACC), areas in the right orbitofrontal cortex, areas in the left hippocampus, midbrain areas involving the bilateral substantia nigra, ventral tegmental area, periaqueductal gray and reticular formation, ventral anterior cingulate cortex (vACC), and parts of the left putamen and left pallidum. The color intensity represents the $T$ value. The color intensity represents the $T$ value.

TABLE 4: SDAEWMT-related decrease in MD compared with the control group.

\begin{tabular}{lcccccc}
\hline \multirow{2}{*}{ Area } & \multicolumn{2}{c}{ MNI coordinates } & \multirow{2}{*}{$T$ score } & \multirow{2}{*}{ Corrected $P$ value (cluster) } \\
& $x$ & $y$ & $z$ & & \multirow{2}{*}{ Cluster size } \\
\hline Midbrain (B)/hippocampus (L)/putamen (L)/pallidum (L) & -13.5 & -16.5 & -13.5 & 5.18 & $<0.001$ & 3071 \\
Middle frontal gyrus (L) & -34.5 & 9 & 46.5 & 4.18 & 0.014 & 901 \\
Superior frontal gyrus (R)/dorsal anterior cingulate cortex (R) & 13.5 & 40.5 & 33 & 3.96 & 0.002 & 1337 \\
Orbital frontal gyrus (R)/ventral anterior cingulate cortex (R) & 12 & 30 & -9 & 3.85 & 0.003 & 1185 \\
\hline
\end{tabular}

MNI: Montreal Neurological Institute.

which consisted of the battery for global executive function, compared with the other two training groups. This result is consistent with a previous review suggesting that simultaneously performed dual-task training incorporating physical training is effective in improving subject performance in this type of general cognitive battery [24]. However, there were no other cognitive functions that showed such patterns. Furthermore, SDAEWMT did not appear to facilitate learning processes for WMT performance, as indicated by the lack of significantly better performance of training tasks compared with the WMT group. We obtained this result despite previous studies reporting that aerobic exercise facilitates learning processes in mice [67]. The reason for this lack of facilitation and for the lack of broad transfer to a wide range of cognitive functions remains unclear. However, as described below, at least some of the WMT tasks employed in this study may have been too difficult for the older adults in our subject cohort. Furthermore, the simultaneously performed dual-task setting may have made it difficult for the exercising subjects to simultaneously focus on the cognitive training tasks. However, these are only speculations, and future studies are required to establish whether SDAEWMT can enhance cognitive training learning processes under different conditions.

The observed MD changes in the SDAEWMT group were found in parts of three major dopaminergic systems. ACC and DLPFC are both involved in top-down attentional control and dual-task execution $[7,68]$, while the hippocampus is involved in long-term memory [69]. On the other hand, the majority of areas where the effects of SDAEWMT 


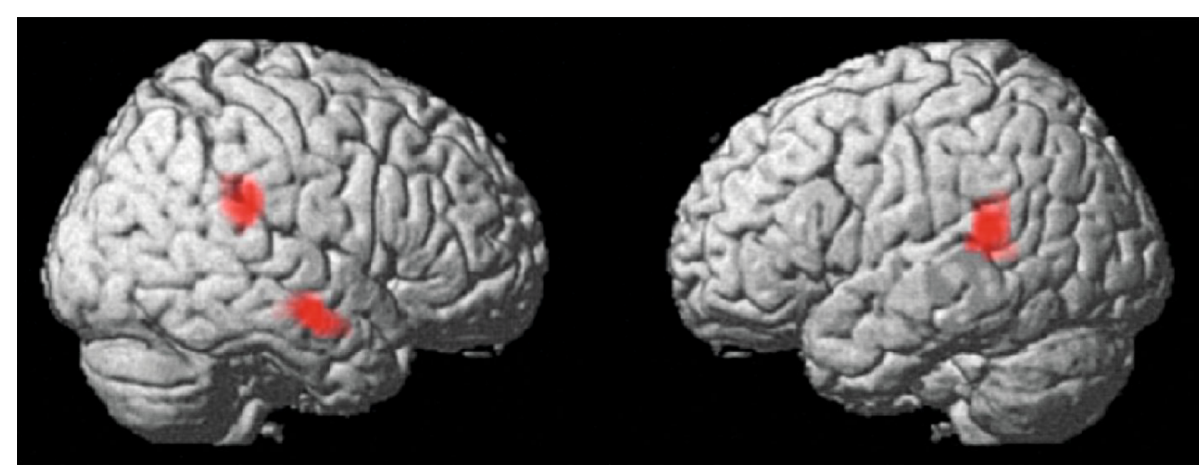

FIGURE 6: Brain activity changes related to training. Increase in brain activity during the 2-back WM task in the SDAEWMT group compared with that in the WMT and AET groups. The results are shown with a threshold of $P<0.05$, corrected for multiple comparisons at the cluster level, with an underlying voxel-level $P<0.001$, uncorrected. Compared with AET, WMT training resulted in increased brain activity during the 2-back WM task in bilateral areas of the temporoparietal junction. The color intensity represents the $T$ value.

on MD were observed are included in the three main dopaminergic systems of the brain. One of these systems is the nigrostriatal system, which projects from the substantia nigra to the striatum and is involved in motor control as well as some higher cognitive functions [70]. The second system is the mesolimbic system, which projects from the ventral tegmental area to regions such as the hippocampus and nucleus accumbens, and is involved in the reward system [70]. The third system is the mesocortical system, which projects from the ventral tegmental area to the prefrontal cortex and is involved in WM and other higher-order cognitive functions [70]. The notion that the dopaminergic system plays at least a partial role in simultaneously performed dual tasks involving physical movement has been suggested by research on Parkinson's disease, which is characterized by degeneration of the dopaminergic system and deficits in such dual-task performance [71]. In addition, while coordination of multiple cognitive processes is assumed to be important for WM [72], as already described, different types of WMT have been shown to affect the dopaminergic system in the frontalparietal areas and the striatum $[73,74]$. Lower MD is known to reflect higher tissue densities resulting from various conditions [50], and this measure is reported to be sensitive to usedependent neural plasticity [51]. However, recent studies have shown that MD in the dopaminergic system is uniquely related to pathological, pharmacological, and cognitive differences or changes directly related to dopamine $[15,75-$ 77]. The changes in MD observed in the present study may reflect alterations in the dopaminergic functions of the three dopaminergic systems. Through these changes, effective dual-task performance and improved frontal lobe functions may be possible. However, future physiological or imaging studies using positron emission topography are required for confirmation.

Functional imaging analysis of WM-related brain activity revealed that SDAEWMT increased WM-related brain activity in areas mainly located around the bilateral temporoparietal junctions, which function in attentional mechanisms. These regions are critical areas of the ventral attention system [78] and are involved in reorienting attention to an outside event or switching attention between different matters [78]. This region is typically deactivated during WM task perfor- mance, potentially to prevent attention from reorienting to distracting events [78]. The elderly can learn to control attention and perform dual task efficiently as they are prepared for simultaneously performed dual-task training involving exercise [79]. Also, a dual-task process typically requires task switching [80]. We believe that the same process partially underlies how the elderly exhibited improved performance in both aerobic capacity and training tasks. Thus, the fact that activity in this region increased following SDAEWMT suggests that through this training, the subjects were able to focus better on outside events or to switch between different tasks. Through these changes, the effective execution of simultaneously performed dual tasks during SDAEWMT may become possible. However, this is speculation based on reverse inference. Future studies are required to elucidate the changes in attentional mechanism induced by SDAEWMT.

The successful implementation of simultaneously performed dual-task training involving exercise in the present study may have implications in daily life. As described in the Introduction, both AET and simultaneously performed dual-task training involving exercise have been shown to be effective in the elderly. However, there is generally a fall risk in the elderly when performing tasks that involve locomotion [81]. In addition, it is difficult to perform these WMTs in daily life while walking or running. Thus, the use of a recumbent bike may be applicable to a wide range of cognitive activities for the elderly in addition to the present dualtraining regimen and may make a wide range of ecologically valid and safe training regimens possible.

The reasons for the lack of significant results in rGMV and FA analyses despite significant effects of WMT on those measures in young adults [14] are not clear. However, previous studies generally suggest the reduction of neural plasticity in the elderly [82], and also, a previous study showed that 1-year AET led to greater rGMV increases than 6-month $\mathrm{AET}$ in some of the brain regions [83]. The length of training might not be long enough to obtain significant results among the different intervention methods in the rGMV analyses.

There are several limitations to this study. First, we could not include passive or active control groups that would have revealed null effects in any of the measures because of our 
resource limitations. Accordingly, we could not detect potential common effects among the three groups, although this design can test the hypothesis that simultaneously combined physical and cognitive training would be more effective than single physical or single cognitive training, as was done in an earlier review [26]. In particular, we could not identify any significant effects of training on digit span or divergent thinking performance This lack of significance may be unexpected given previous studies reporting consistent WMT effects on untrained WM performance, including effects of $N$-back task training on digit span performance $[11,84]$ and the consistently reported effects of AET on divergent thinking performance [85]. However, a previous three-month intervention study involving older adults also did not show any increase in digit span performance in two control groups $(0.3$ and 0 point decreases from pre- to posttest, respectively) [86]. Furthermore, in our studies on young adults, including two separate one-month intervention studies, S-A creativity test performance did not show a mean increase in the passive control group [66]. On the other hand, the 93 subjects who completed the present intervention showed an increase in digit span performance (1.194 point increase, $P=5.473 \times$ $10-15$, paired $t$-test) and S-A creativity test performance ( 1.527 point increase, $P=1.931 \times 10-18$, paired $t$-test). In the present study, the game used for WMT was apparently too difficult for the older subjects to complete. In particular, in each of the three $N$-back WMT tasks, the final performance level was only slightly different from the lowest level for the WM condition (2-back task). The initial performance levels for these tasks were also low, and based on nonquantitative observation of training, the subjects appeared to have difficulty in adopting an effective strategy for completing the task, consistent with previous reports [87]. These observations reveal a less effective adaptive difficulty control (i.e., when the span increases from 3 to 4 , the level increases by $33 \%$, which is twice as large as when the span increases from 6 to 7 ), resulting in only modest improvement in tasks such as the digit span. Future studies evaluating young adult subjects or incorporating easier WMT tasks for older adults may be warranted to address this problem. Further, in this study, measurements of cardiovascular fitness were not performed postexperiment as they were not central to the purpose of the study and as it takes resources to perform the procedures. HRRSBE was gathered, but only from participants in the AET and SDAEWMT groups. Thus, the effects of aerobic exercise might not have been sufficiently evaluated in this study. Also, in the AET and SDAEWMT groups, participants could select the order of tasks that they conducted during the training sessions. Although this procedure might mitigate the toughness of training, it might increase the unwanted variables among training groups and reduce the sensitivity of the analysis. Further, there is a lack of follow-up experimentation after the training ceased, to evaluate how long the training effects lasted; this lack is due to the practical limitation of the various kinds of resources. Intervention studies in the field often take place without an opportunity to evaluate the duration of the training effects, and future studies should investigate this aspect. Finally, all the subjects were asked to engage in training three times each week in a laboratory, but if a subject was not available during a given week, he/she was allowed to make up for the lost sessions in the following weeks. This was necessary as participants have daily lives that do not always fit the laboratory schedule. This kind of imperfect control of the training protocol might affect the findings.

From the present results, we showed the effects of SDAEWMT and made the following conclusions: (a) SDAEWMT improved executive function in the frontal lobe. (b) There was no evidence of broader transfer effects such as in memory performance and attention measures (in contrast to our hypothesis) or in processing speed, divergent thinking, or other effects that enhanced learning. (c) SDAEWMT resulted in neural tissue changes in brain areas involving the dopaminergic system. (d) SDAEWMT produced an increase in WM-related brain activity in brain areas involved in attentional reorienting.

\section{Data Availability}

All the experimental data obtained in the experiment of this study will be available to ones that were admitted in the ethics committee of Tohoku University, school of medicine. All the data sharing should be first admitted by the ethics committee of Tohoku University, school of medicine.

\section{Ethical Approval}

All procedures performed in studies involving human participants were in accordance with the ethical standards of the institutional and/or national research committee and with the 1964 Helsinki declaration and its later amendments or comparable ethical standards. This study was approved by the Ethics Committee of Tohoku University.

\section{Consent}

Informed consent was obtained from all individual participants included in the study.

\section{Conflicts of Interest}

RK is the creator of Brain Age: Concentration Training. Tohoku University, to which RK belongs, received royalties generated by Brain Age: Concentration Training's sales. RK has no other competing interests. The research fund from Nintendo, which sold Brain Age: Concentration Training, was used for this study. All other authors have declared that no competing interests exist.

\section{Authors' Contributions}

Hikaru Takeuchi and Daniele Magistro contributed equally to this work.

\section{Acknowledgments}

We thank Yuki Yamada for operating the MRI scanner; all our other colleagues in IDAC, Tohoku University for their support; Kuniyoshi Sugimura, Shun-ichi Sone, and all staff of Ichinazaka medical clinic for conducting the health 
assessment to potential subjects, and nurses in Sendai city for overseeing the aerobic exercise. We would like to thank Dr. Takamitsu Shinada for his help in conducting the experiment. This study was supported by JST/RISTEX, JST/CREST, the JSPS Postdoctoral Fellowship Programme for Foreigner Researchers FY2012, and the Naito Memorial Foundation. This research has been partially supported by the JSPS Grant-in-Aid for Young Scientists (Wakate B) (25750259 and 15K21437).

\section{Supplementary Materials}

Supplementary online material consists of Supplemental Methods which describe exclusion criteria of the subjects, details of neuropsychological tests, and details of each training task. (Supplementary Materials)

\section{References}

[1] World Health Organization, Global Health Observatory Data Repository (2014) Health expenditure ratios: data by country, Series Editor, 2015.

[2] S. A. P. Clouston, P. Brewster, D. Kuh et al., "The dynamic relationship between physical function and cognition in longitudinal aging cohorts," Epidemiologic Reviews, vol. 35, no. 1, pp. 33-50, 2013.

[3] C. Lustig, P. Shah, R. Seidler, and P. A. Reuter-Lorenz, "Aging, training, and the brain: a review and future directions," Neuropsychology Review, vol. 19, no. 4, pp. 504-522, 2009.

[4] G. Yogev-Seligmann, J. M. Hausdorff, and N. Giladi, "The role of executive function and attention in gait," Movement Disorders, vol. 23, no. 3, pp. 329-342, 2008.

[5] W. J. Chodzko-Zajko, "Exercise and physical activity for older adults," Medicine \& Science in Sports \& Exercise, vol. 41, no. 7, pp. 1510-1530, 1998.

[6] A. Baddeley, "Working memory: looking back and looking forward," Nature Reviews Neuroscience, vol. 4, no. 10, pp. 829-839, 2003.

[7] M. Corbetta and G. L. Shulman, "Control of goal-directed and stimulus-driven attention in the brain," Nature Reviews Neuroscience, vol. 3, no. 3, pp. 201-215, 2002.

[8] A. R. Laird, K. M. McMillan, J. L. Lancaster et al., "A comparison of label-based review and ALE meta-analysis in the Stroop task," Human Brain Mapping, vol. 25, no. 1, pp. 6-21, 2005.

[9] R. L. Buckner, J. R. Andrews-Hanna, and D. L. Schacter, "The Brain's Default Network," Annals of the New York Academy of Sciences, vol. 1124, no. 1, pp. 1-38, 2008.

[10] S. Aalto, A. Bruck, M. Laine, K. Nagren, and J. O. Rinne, "Frontal and temporal dopamine release during working memory and attention tasks in healthy humans: a positron emission tomography study using the high-affinity dopamine D2 receptor ligand [11C] FLB 457," Journal of Neuroscience, vol. 25, no. 10, pp. 2471-2477, 2005.

[11] M. Melby-Lervåg and C. Hulme, "Is working memory training effective? A meta-analytic review," Developmental Psychology, vol. 49, no. 2, pp. 270-291, 2013.

[12] J. Au, E. Sheehan, N. Tsai, G. J. Duncan, M. Buschkuehl, and S. M. Jaeggi, "Improving fluid intelligence with training on working memory: a meta-analysis," Psychonomic Bulletin and Review, vol. 22, no. 2, pp. 1-12, 2014.
[13] J. Au, M. Buschkuehl, G. J. Duncan, and S. M. Jaeggi, “There is no convincing evidence that working memory training is NOT effective: a reply to Melby-Lervåg and Hulme (2015)," Psychonomic Bulletin and Review, vol. 23, no. 1, pp. 331-337, 2016.

[14] H. Takeuchi, Y. Taki, and R. Kawashima, "Effects of working memory training on cognitive functions and neural systems," Reviews in the Neurosciences, vol. 21, no. 6, pp. 427-449, 2010.

[15] H. Takeuchi, Y. Taki, R. Nouchi et al., "Working memory training impacts the mean diffusivity in the dopaminergic system," Brain Structure and Function, vol. 220, no. 6, pp. 31013111, 2015.

[16] T. Klingberg, "Training and plasticity of working memory," Trends in Cognitive Sciences, vol. 14, no. 7, pp. 317-324, 2010.

[17] L. Backman, L. Nyberg, A. Soveri et al., "Effects of workingmemory training on striatal dopamine release," Science, vol. 333, no. 6043, pp. 718-718, 2011.

[18] F. Candela, G. Zucchetti, D. Magistro, and E. Rabaglietti, “The effects of a physical activity program and a cognitive training program on the long-term memory and selective attention of older adults: a comparative study," Activities, Adaptation \& Aging, vol. 39, no. 1, pp. 77-91, 2015.

[19] P. J. Smith, J. A. Blumenthal, B. M. Hoffman et al., "Aerobic exercise and neurocognitive performance: a meta-analytic review of randomized controlled trials," Psychosomatic Medicine, vol. 72, no. 3, pp. 239-252, 2010.

[20] A. Rathore and B. Lom, "The effects of chronic and acute physical activity on working memory performance in healthy participants: a systematic review with meta-analysis of randomized controlled trials," Systematic reviews, vol. 6, no. 1, p. 124, 2017.

[21] P. D. Gajewski and M. Falkenstein, "ERP and behavioral effects of physical and cognitive training on working memory in aging: a randomized controlled study," Neural Plasticity, vol. 2018, Article ID 3454812, 12 pages, 2018.

[22] C. H. Hillman, K. I. Erickson, and A. F. Kramer, "Be smart, exercise your heart: exercise effects on brain and cognition," Nature Reviews Neuroscience, vol. 9, no. 1, pp. 58-65, 2008.

[23] A. Baddeley, S. Della Sala, C. Papagno, and H. Spinnler, "Dualtask performance in dysexecutive and nondysexecutive patients with a frontal lesion," Neuropsychology, vol. 11, no. 2, pp. 187-194, 1997.

[24] L. L. F. Law, F. Barnett, M. K. Yau, and M. A. Gray, "Effects of combined cognitive and exercise interventions on cognition in older adults with and without cognitive impairment: a systematic review," Ageing Research Reviews, vol. 15, pp. 61-75, 2014.

[25] K. I. Erickson, S. J. Colcombe, R. Wadhwa et al., "Traininginduced functional activation changes in dual-task processing: an FMRI study," Cerebral Cortex, vol. 17, no. 1, pp. 192-204, 2007.

[26] A. Lauenroth, A. E. Ioannidis, and B. Teichmann, "Influence of combined physical and cognitive training on cognition: a systematic review," BMC Geriatrics, vol. 16, no. 1, 2016.

[27] T. Klingberg, H. Forssberg, and H. Westerberg, "Training of working memory in children with ADHD," Journal of Clinical and Experimental Neuropsychology, vol. 24, no. 6, pp. 781-791, 2010.

[28] R. C. Oldfield, "The assessment and analysis of handedness: the Edinburgh inventory," Neuropsychologia, vol. 9, no. 1, pp. 97-113, 1971.

[29] M. J. Karvonen, "The effects of training on heart rate. A longitudinal study," Annales Medicinae Experimentalis et Biologiae Fenniae, vol. 35, no. 3, pp. 307-315, 1957. 
[30] H. Tanaka, K. D. Monahan, and D. R. Seals, "Age-predicted maximal heart rate revisited," Journal of the American College of Cardiology, vol. 37, no. 1, pp. 153-156, 2001.

[31] J. L. Etnier, W. Salazar, D. M. Landers, S. J. Petruzzello, M. Han, and P. Nowell, "The influence of physical fitness and exercise upon cognitive functioning: a meta-analysis," Journal of Sport and Exercise Psychology, vol. 19, no. 3, pp. 249-277, 1997.

[32] C. Andy, T. W. Wong, and P. H. Lee, "Effect of low-intensity exercise on physical and cognitive health in older adults: a systematic review," Sports medicine-open, vol. 1, no. 1, 2015.

[33] J. S. Stevenson and R. Topp, "Effects of moderate and low intensity long-term exercise by older adults," Research in Nursing and Health, vol. 13, no. 4, pp. 209-218, 1990.

[34] S. Colcombe and A. F. Kramer, "Fitness effects on the cognitive function of older adults: a meta-analytic study," Psychological Science, vol. 14, no. 2, pp. 125-130, 2016.

[35] J. Raven, Manual for Raven's Progressive Matrices and Vocabulary Scales, Oxford Psychologists Press, Oxford, 1998.

[36] M. Sugishita, Wechsler Memory Scale-Revised Japanese Edition, Nihon bunka kagakusya, Tokyo, 2002.

[37] T. Hatta, Y. Ito, and K. Yoshizaki, D-CAT Manual (Screening Test for Attention), Union Press, Osaka, 2000.

[38] B. Dubois, A. Slachevsky, I. Litvan, and B. Pillon, "The FAB a frontal assessment battery at bedside," Neurology, vol. 55, no. 11, pp. 1621-1626, 2000.

[39] R. Nouchi, Y. Taki, H. Takeuchi et al., "Brain training game improves executive functions and processing speed in the elderly: a randomized controlled trial," PLoS ONE, vol. 7, no. $1,2012$.

[40] D. Wechsler, Wechsler adult intelligence scale, The Psychological Corporation, New York, 1997.

[41] Society For Creative Minds, Manual of S-A creativity test, Tokyo shinri Corporation, Tokyo, Japan, 1969.

[42] H. Takeuchi, Y. Taki, Y. Sassa et al., "White matter structures associated with creativity: evidence from diffusion tensor imaging," NeuroImage, vol. 51, no. 1, pp. 11-18, 2010.

[43] H. Takeuchi, Y. Taki, Y. Sassa et al., "Regional gray matter volume of dopaminergic system associate with creativity: evidence from voxel-based morphometry," NeuroImage, vol. 51, no. 2, pp. 578-585, 2010.

[44] M. F. Folstein, S. E. Folstein, and P. R. McHugh, ““Mini-mental state": a practical method for grading the cognitive state of patients for the clinician," Journal of Psychiatric Research, vol. 12, no. 3, pp. 189-198, 1975.

[45] Y. Hakoda and M. Sasaki, "Group version of the Stroop and reverse-Stroop test : the effects of reaction mode, order and practice," Kyoikushinrigakukenkyu (Educational Psychology Research), vol. 38, no. 4, pp. 389-394, 1990.

[46] H. Takeuchi, M. Sugiura, Y. Sassa et al., "Neural correlates of the difference between working memory speed and simple sensorimotor speed: an fMRI study," PLoS ONE, vol. 7, no. 1, p. e30579, 2012.

[47] H. Takeuchi, Y. Taki, H. Hashizume et al., "Effects of training of processing speed on neural systems," Journal of Neuroscience, vol. 31, no. 34, pp. 12139-12148, 2011.

[48] J. Jansma, N. Ramsey, N. Van Der Wee, and R. Kahn, "Working memory capacity in schizophrenia: a parametric fMRI study," Schizophrenia Research, vol. 68, no. 2-3, pp. 159-171, 2004.
[49] S. Whitfield-Gabrieli, H. W. Thermenos, S. Milanovic et al., "Hyperactivity and hyperconnectivity of the default network in schizophrenia and in first-degree relatives of persons with schizophrenia," Proceedings of the National Academy of Sciences of the United States of America, vol. 106, no. 4, pp. 1279-1284, 2009.

[50] C. Beaulieu, "The basis of anisotropic water diffusion in the nervous system-a technical review," NMR in Biomedicine, vol. 15, no. 7-8, pp. 435-455, 2002.

[51] Y. Sagi, I. Tavor, S. Hofstetter, S. Tzur-Moryosef, T. Blumenfeld-Katzir, and Y. Assaf, "Learning in the fast lane: new insights into neuroplasticity," Neuron, vol. 73, no. 6, pp. 1195-1203, 2012.

[52] H. Takeuchi, A. Sekiguchi, Y. Taki et al., "Training of working memory impacts structural connectivity," Journal of Neuroscience, vol. 30, no. 9, pp. 3297-3303, 2010.

[53] T. Salminen, J. Mårtensson, T. Schubert, and S. Kühn, "Increased integrity of white matter pathways after dual nback training," NeuroImage, vol. 133, pp. 244-250, 2016.

[54] J. Diedrichsen and R. Shadmehr, "Detecting and adjusting for artifacts in fMRI time series data," NeuroImage, vol. 27, no. 3, pp. 624-634, 2005.

[55] J. Ashburner and G. R. Ridgway, "Symmetric diffeomorphic modeling of longitudinal structural MRI," Frontiers in Neuroscience, vol. 6, 2013.

[56] H. Takeuchi, Y. Taki, R. Nouchi et al., "Effects of multitaskingtraining on gray matter structure and resting state neural mechanisms," Human Brain Mapping, vol. 35, no. 8, pp. 3646-3660, 2014.

[57] D. M. Dimitrov, J. Rumrill, and D. Phillip, "Pretest-posttest designs and measurement of change," Work, vol. 20, no. 2, pp. 159-165, 2003.

[58] H. Takeuchi, Y. Taki, Y. Sassa et al., "Working memory training using mental calculation impacts regional gray matter of the frontal and parietal regions," PLoS ONE, vol. 6, no. 8, p. e23175, 2011.

[59] T. Klingberg, E. Fernell, P. J. Olesen et al., "Computerized training of working memory in children with ADHD-a randomized, controlled trial," Journal of the American Academy of Child and Adolescent Psychiatry, vol. 44, no. 2, pp. 177186, 2005.

[60] Y. Benjamini, A. M. Krieger, and D. Yekutieli, “Adaptive linear step-up procedures that control the false discovery rate," Biometrika, vol. 93, no. 3, pp. 491-507, 2006.

[61] R. Casanova, R. Srikanth, A. Baer et al., "Biological parametric mapping: a statistical toolbox for multimodality brain image analysis," NeuroImage, vol. 34, no. 1, pp. 137-143, 2007.

[62] J. H. Callicott, V. S. Mattay, A. Bertolino et al., "Physiological characteristics of capacity constraints in working memory as revealed by functional MRI," Cerebral Cortex, vol. 9, no. 1, pp. 20-26, 1999.

[63] K. J. Friston, A. Holmes, J. B. Poline, C. J. Price, and C. D. Frith, "Detecting activations in PET and fMRI: levels of inference and power," NeuroImage, vol. 4, no. 3, pp. 223-235, 1996.

[64] S. M. Smith and T. E. Nichols, "Threshold-free cluster enhancement: addressing problems of smoothing, threshold dependence and localisation in cluster inference," NeuroImage, vol. 44, no. 1, pp. 83-98, 2009.

[65] S. Hayasaka, K. L. Phan, I. Liberzon, K. J. Worsley, and T. E. Nichols, "Nonstationary cluster-size inference with random 
field and permutation methods," NeuroImage, vol. 22, no. 2, pp. 676-687, 2004.

[66] H. Takeuchi, Y. Taki, R. Nouchi et al., "Effects of working memory-training on functional connectivity and cerebral blood flow during rest," Cortex, vol. 49, no. 8, pp. 2106-2125, 2013.

[67] M. L. Mustroph, S. Chen, S. C. Desai, E. B. Cay, E. K. DeYoung, and J. S. Rhodes, "Aerobic exercise is the critical variable in an enriched environment that increases hippocampal neurogenesis and water maze learning in male C57BL/6J mice," Neuroscience, vol. 219, pp. 62-71, 2012.

[68] A. J. Szameitat, T. Schubert, K. Müller, and D. Y. von Cramon, "Localization of executive functions in dual-task performance with fMRI," Journal of Cognitive Neuroscience, vol. 14, no. 8, pp. 1184-1199, 2002.

[69] S. Köhler, S. E. Black, M. Sinden et al., "Memory impairments associated with hippocampal versus parahippocampal-gyrus atrophy: an MR volumetry study in Alzheimer's disease," Neuropsychologia, vol. 36, no. 9, pp. 901-914, 1998.

[70] N. R. Carlson, Physiology of Behavior, Allyn and Bacon, Boston, 2001.

[71] V. E. Kelly, A. J. Eusterbrock, and A. Shumway-Cook, "A Review of Dual-Task Walking Deficits in People with Parkinson's Disease: Motor and Cognitive Contributions, Mechanisms, and Clinical Implications," Parkinson's Disease, vol. 2012, Article ID 918719, 14 pages, 2012.

[72] A. D. Baddeley and S. Della Sala, "Working memory and executive control," Philosophical Transactions of the Royal Society of London Series B: Biological Sciences, vol. 351, no. 1346, pp. 1397-1404, 1996.

[73] F. McNab, A. Varrone, L. Farde et al., "Changes in cortical dopamine D1 receptor binding associated with cognitive training," Science, vol. 323, no. 5915, pp. 800-802, 2009.

[74] L. Bäckman and L. Nyberg, "Dopamine and training-related working-memory improvement," Neuroscience \& Biobehavioral Reviews, vol. 37, no. 9, pp. 2209-2219, 2013.

[75] H. Takeuchi, Y. Taki, A. Sekiguchi et al., "Mean diffusivity of globus pallidus associated with verbal creativity measured by divergent thinking and creativity-related temperaments in young healthy adults," Human Brain Mapping, vol. 36, no. 5, pp. 1808-1827, 2015.

[76] A. A. Razek, A. Elmongy, M. Hazem, S. Zakareyia, and W. Gabr, "Idiopathic Parkinson disease effect of levodopa on apparent diffusion coefficient value of the brain," Academic Radiology, vol. 18, no. 1, pp. 70-73, 2011.

[77] P. Péran, A. Cherubini, F. Assogna et al., "Magnetic resonance imaging markers of Parkinson's disease nigrostriatal signature," Brain, vol. 133, no. 11, pp. 3423-3433, 2010.

[78] M. Corbetta, G. Patel, and G. L. Shulman, "The reorienting system of the human brain: from environment to theory of mind," Neuron, vol. 58, no. 3, pp. 306-324, 2008.

[79] L. Bherer, A. F. Kramer, M. S. Peterson, S. Colcombe, K. Erickson, and E. Becic, "Transfer effects in task-set cost and dual-task cost after dual-task training in older and younger adults: further evidence for cognitive plasticity in attentional control in late adulthood," Experimental Aging Research, vol. 34, no. 3, pp. 188-219, 2008.

[80] I. Koch, E. Poljac, H. Müller, and A. Kiesel, “Cognitive structure, flexibility, and plasticity in human multitasking - an integrative review of dual-task and task-switching research," Psychological Bulletin, vol. 144, no. 6, pp. 557-583, 2018.
[81] S. Springer, N. Giladi, C. Peretz, G. Yogev, E. S. Simon, and J. M. Hausdorff, "Dual-tasking effects on gait variability: the role of aging, falls, and executive function," Movement Disorders, vol. 21, no. 7, pp. 950-957, 2006.

[82] E. Dahlin, A. S. Neely, A. Larsson, L. Backman, and L. Nyberg, "Transfer of learning after updating training mediated by the striatum," Science, vol. 320, no. 5882, pp. 1510-1512, 2008.

[83] K. I. Erickson, M. W. Voss, R. S. Prakash et al., "Exercise training increases size of hippocampus and improves memory," Proceedings of the National Academy of Sciences, vol. 108, no. 7, pp. 3017-3022, 2011.

[84] S. M. Jaeggi, M. Buschkuehl, J. Jonides, and W. J. Perrig, "Improving fluid intelligence with training on working memory," Proceedings of the National Academy of Sciences of the United States of America, vol. 105, no. 19, pp. 6829-6833, 2008.

[85] H. Steinberg, E. A. Sykes, T. Moss, S. Lowery, N. LeBoutillier, and A. Dewey, "Exercise enhances creativity independently of mood," British Journal of Sports Medicine, vol. 31, no. 3, pp. 240-245, 1997.

[86] H. W. Mahncke, B. B. Connor, J. Appelman et al., "Memory enhancement in healthy older adults using a brain plasticitybased training program: a randomized, controlled study," Proceedings of the National Academy of Sciences of the United States of America, vol. 103, no. 33, pp. 12523-12528, 2006.

[87] G. Brébion, M. Smith, and M. Ehrlich, "Working memory and aging: deficit or strategy differences?," Aging, Neuropsychology, and Cognition, vol. 4, no. 1, pp. 58-73, 1997. 